\title{
Commentary: "The Development of an Ideal Antibiotic Compound: A Fairy Tale or a Possible Reality?"
}

\author{
Márió Gajdács \\ Department of Pharmacodynamics and Biopharmacy, Faculty of Pharmacy, University of Szeged, Szeged, Hungary
}

Article Info

Article Notes

Received: April 8, 2019

Accepted: April 28, 2019

\section{${ }^{*}$ Correspondence:}

Márió Gajács, Department of Pharmacodynamics and Biopharmacy, Faculty of Pharmacy, University of Szeged, Szeged, Hungary; Email: gajdacs.mario@pharm.u-szeged.hu.

C 2019 Gajdács M. This article is distributed under the terms of the Creative Commons Attribution 4.0 International License.

\section{Keywords}

Antibiotic

Multidrug-resistance

Drug discovery

ESKAPE

Prodrug

Persisters

\section{Original article citation:}

"The concept of an ideal antibiotic: implications for drug design" by Márió Gajdács. Molecules 2019, 24(5), 892; doi: 10.3390/molecules 24050892
After the discovery of penicillin by Alexander Flemming in 1928, the landscape of healthcare has changed drastically, and previously lethal infections have become treatable ${ }^{1}$. However, the emergence and spread of multidrug-resistant bacteria is currently a major public health issue affecting patients, healthcare professionals, scientists, drug development companies and government officials alike $^{2}$. Various bacterial resistance mechanisms have been described, allowing pathogens to evade lethal effects of antibiotics, the most important mechanisms being enzymatic degradation (e.g., $\quad \beta$-lactamases, aminoglycoside-degrading enzymes), target alteration (e.g., penicillin-binding proteins, bacterial topoisomerases), decreased uptake (porin-deficient mutants) and overexpression of energy-dependent efflux pump proteins (e.g., AcrAB-TolC in Enterobacteriaceae) ${ }^{3}$. Multidrug-resistant (MDR) bacteria can withstand potentially lethal doses of antibiotics with various chemical structures and mechanisms of action. The World Health Organization (WHO), the European Center for Disease Prevention and Control (ECDC), and the Centers for Disease Control and Prevention in the US (CDC) have all published reports on the significance of MDR bacteria ${ }^{4}$. All of these reports concluded that antibiotic resistance is a global issue that may become the major cause of mortality by 2050. So-called "ESKAPE" bacteria have been described as the most important problem, including E: Enterococcus faecium, S: Staphylococcus aureus or recently Stenotrophomonas maltophilia, K: Klebsiella pneumoniae or recently C: Clostridioides difficile, A: Acinetobacter baumannii, P: Pseudomonas aeruginosa, E: Enterobacter spp., or recently Enterobacteriaceae ${ }^{5}$.

The speed of the discovery of novel antibiotics is no match for the continuous and detrimental changes in resistance trends. In the "golden age" of antibiotic discovery (1950-1980), there were similar developments in bacterial resistance; but the newly developed drugs or structurally-modified active derivatives of old drugs had adequate activity ${ }^{6}$. This resulted in a shift in interest towards the treatment of chronic illnesses by pharmaceutical companies, slowing down development of new antibacterial drugs. Unfortunately, after the introduction of fluoroquinolones in the 1960s, no broad-spectrum agents have been discovered: linezolid and daptomycin are only useful in the treatment of Gram-positive infections (e.g. S. aureus or Enterococcus spp., while polymixins (cyclic polypeptides with pronounced toxicity), were only recently re-introduced to therapy, due to the increasing prevalence of drug resistant Gram-negative pathogens. In addition, many pharmaceutical companies have left antimicrobial research, such as Novartis, AstraZeneca, Sanofi, 
Bristol-Myers Squibb, and Allergan 7 . Because there is a very low probability for biologically active compounds to successfully emerge from the pre-clinical studies, reliable discovery platforms are needed to produce compounds with antibacterial activity that may be lead compounds for further studies. Previously efficient antibiotic discovery platforms (Domagk-platform/ in situ screening-platform, Waksman-platform/natural products-platform, speciesselective platform, high-throughput screening (HTS)/ rational drug discovery) did not survive the test of time when it comes to churning out new antimicrobial drugs, while emerging discovery pathways did not yet produce lead compounds for clinical use (e.g., antimicrobial peptides (AMPs), resistance reversing compounds, virulence modulatory compounds) ${ }^{8}$. In addition to discovery platforms, it would be useful to hypothesize about a concept of an ideal antibiotic, with the intention to direct antibacterial discovery and drug design, and to aid researchers in selecting promising lead compounds.

This ideal antibiotic should have the following properties (discussed in detail in Gajdács et al., 20197): broad-spectrum bactericidal activity against metabolically active and dormant/persisting bacteria, in addition to pathogens hiding in biofilms. The compounds should also be resistant to efflux pump-based resistance, by forming an irreversible, covalent bond on multiple, unrelated targets, leading to bacterial cell death. An emerging concept is that the molecule should function as a prodrug. In addition to the interactions of the molecule with the target microorganisms, these compounds must meet a set of predetermined characteristics to be appropriate in clinical use. They should be used orally ( $>90 \%$ bioavailability), comply with Lipinsky's Rule of Five (R05) and be a Class I in the Biopharmaceutical Classification System. The compounds should be able to accumulate in macrophages and non-professional phagocytes which is relevant in the elimination of obligate and facultative intracellular bacteria. An ideal drug would be useful in vulnerable patient populations such as pregnant and lactating women, as well as in children and the elderly. Ideally, an antibiotic should be metabolized without affecting liver enzymes and it should be eliminated without alteration from the body (e.g., in the urine).

One can wonder whether the synthesis and production of the ideal antibiotic is realistic, or if this will only remain a theoretical concept or if it would be realistic to identify and design such molecules. Actually, there are a few drugs in current clinical use that have similar characteristics to this model, namely ethionamide, isoniazid, pyrazinamide and the metronidazole-like drugs. Metronidazole act as a prodrug, and it must be reduced by specific enzymes (namely nitro-reductases and redox-active enzymes, such as pyruvate:ferredoxin/pyruvate:flavodoxin oxidoreductase and hydrogenase) to produce highly reactive species, damaging the bacterial cell membrane, DNA and proteins". Ethionamide, isoniazid, and pyrazinamide are all drugs that are relevant in the treatment of $\mathrm{TB}^{10}$. All three drugs are bactericidal, and they can penetrate well into macrophages ${ }^{10}$. They also turn into active derivatives after activation by EthA (a flavin mono-oxygenase)/KatG (a catalase-peroxidase)/pncA (PZase/nicotinamidase) ${ }^{10}$. It's not surprising that the above-mentioned drugs are listed in the Essential Medicines List of the World Health Organization, indicating their importance and the need for universal access ${ }^{11}$.

The production of a compounds possessing every single attribute listed in the article is unlikely, however, the usefulness of this model is to aim towards specific features from the list, based on the pathogen, site of infection, administration route, and the targeted patient population during drug development. The modification/ functionalization of existing antibiotics is a more probable approach. Some alternative approaches for combating infectious diseases are being developed, but it is unreasonable to believe that they will replace antibiotics. New energy in developing new classes of antimicrobials, novel developmental strategies (e.g., computer-aided autonomous discovery of peptide antibiotics, the use of data mining for antibiotic discovery, repurposing of toxic molecules into viable antimicrobials etc.) $)^{12-14}$ and the prudent use of existing agents is of utmost importance ${ }^{15,16}$.

\section{Acknowledgments}

M.G. was supported by the National Youth Excellence Scholarship (grant number NTP-NTFÖ-18-C-0225), and the ESCMID Observership Programme.

\section{References}

1. Gaynes R. The Discovery of Penicillin-New Insights after More Than 75 Years of Clinical Use. Emerg Infect Dis. 2017; 23: 849-853.

2. Spellberg B. The future of antibiotics. Crit Care. 2014; 18: 228

3. Spengler G, Kincses A, Gajdacs M, et al. New Roads Leading to Old Destinations: Efflux Pumps as Targets to Reverse Multidrug Resistance in Bacteria. Molecules. 2017; 22: 468.

4. Gajdács M. [Extra deaths due to pandrug resistant bacteria: a survey of the literature] (article in Hungarian). Egészségfejlesztés. 60: 31-36.

5. Santajit S, Indrawattana N. Mechanisms of Antimicrobial Resistance in ESKAPE Pathogens. Biomed Res Int. 2016; 2016: 2475067.

6. Spellberg B. The future of antibiotics. Crit Care. 2014; 18: 228.

7. Gajdács M. The Concept of an Ideal Antibiotic: Implications for Drug Design. Molecules. 2019; 24: 892.

8. Lewis K. Platforms for antibiotic discovery. Nat Rev Drug Discov. 2013; 12: 371-387.

9. Gajdács M, Spengler G, Urbán E. Identification and Antimicrobial Susceptibility Testing of Anaerobic Bacteria: Rubik's Cube of Clinical Microbiology? Antibiotics (Basel). 2017; 6: 25. 
10. Sotgiu G, Centis R, D’ambrosio L, et al. Tuberculosis Treatment and Drug Regimens. Cold Spring Harb Perspect Med. 2015; 5: a017822.

11. The Selection and Use of Essential Medicines. World Health Organization. Available online: https://www.who.int/medicines/ publications/essentialmedicines/EML_2017_ExecutiveSummary. pdf?ua=1 (accessed on 23 April 2019).

12. Porto WF, Irazazabal L, Alves ESF, et al. In silico optimization of a guava antimicrobialpeptide enables combinatorial exploration forpeptide design. Nature Communications. 2018; 9: 1490

13. Katia Pane, Valeria Cafaro, Angela Avitabile, et al. Identification of Novel Cryptic Multifunctional Antimicrobial Peptides from the Human
Stomach Enabled by a Computational-Experimental Platform. ACS Synth Biol. 2018; 7 (9): 2105-2115.

14. Torres MDT, Pedron CN, Higashikuni Y. Structure-function-guided exploration of the antimicrobial peptide polybia-CP identifies activity determinants and generates synthetic therapeutic candidates. ommunications Biology. 2018; 1: 221.

15. Gajdács M, Paulik E, Szabó A. [The attitude of community pharmacists towards their widening roles in the prevention and treatment of infectious diseases in the southeast region of Hungary] (article in Hungarian). Gyógyszerészet. 2019; 63: 26-30.

16. Singh SB, Young K, Silver LL. What is an "ideal" antibiotic? Discovery challenges and path forward. Biochem Pharmacol. 2017; 133: 63-73. 